\title{
Riesgo de enfermedad entre los profesionales de la construcción
}

Risk of illness among construction professionals Risco de adoecimento entre profissionais da construção civil

\begin{abstract}
Priscila Locatelli ${ }^{1}$, Katrini dos Santos Conteratto ${ }^{2}$, Clodoaldo Antônio De Sá $^{3}$, Liana Lautert ${ }^{4}$, Rosana Amora Ascari ${ }^{5}$

${ }^{1}$ Enfermeira. Universidade do Estado de Santa Catarina-UDESC (Brasil). Bolsista PIVIC.Correo electrónico: prii_locatelli@hotmail.com

${ }^{2}$ Acadêmica de Enfermagem da UDESC (Brasil). Bolsista PIVIC. Correo electrónico: katrinii_conteratto@hotmail.com

${ }^{3}$ Doutor em Ciência do Movimento Humano. Coordenador do Programa de Pós-Graduação em Ciências da Saúde da Universidade Comunitária da Região de Chapecó-UNOCHAPECÓ (Brasil). Correo electrónico: clodoaldo@unochapeco.edu.br

${ }^{4}$ Enfermeira. Doutora em Psicologia. Docente da Universidade Federal do Rio Grande do Sul-UFRGS (Brasil).Correo electrónico: lilabem@gmail.com

${ }^{5}$ Doutora em Enfermagem. Docente do Departamento de Enfermagem da Universidade do Estado de santa Catarina-UDESC. Membro do Grupo de Estudos sobre Saúde e Trabalho-Gestra/Udesc (Brasil)

Cómo citar este artículo en edición digital: Locatelli, P., Conteratto, K. S., De Sá, C. A., Lautert, L. E Ascari, R. A. (2019). Riesgo de enfermedad entre los profesionales de la construcción. Cultura de los Cuidados (Edición digital), 23(53). Recuperado de http://dx.doi.org/10.14198/cuid.2019.53.18

Correspondencia: Rosana Amora Ascari. Rua 14 de agosto, 807 E, Bairro Presidente Médici, Chapecó-SC, Brasil. CEP: 89.801-251

Correo electrónico de contacto: rosana.ascari@hotmail.com
\end{abstract}

Recibido: 12/11/2018; Aceptado: 23/01/2019

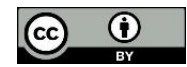

\section{ABSTRACT}

Objective: To evaluate the risks of sickness present in the work activities of construction workers and the factors associated with the risk of illness in this population. Methods: This is a cross-sectional research with a quantitative approach, through the Inventory of Work and Illness Risks. Participated 75 workers linked to a construction company, after approval by the Research Ethics Committee under CAAE n. ${ }^{\text {o }}$ 22445413.3.0000.0118. Results: There was a predominance of males, between 18 and 28 years of age, married, with low schooling, with 6 to 10 years of profession. Emphasis is given to the factors: Conditions of Work, Physical Cost and Lack of Recognition, which had "negative, severe" evaluation, characterizing human cost production and labor suffering.

Results: The results point out risks of illness among construction professionals.

Conclusion: It is necessary to review the working conditions in the scenario investigated in order to reduce the risk of illness among construction workers.

Keywords: Occupational risks, Occupational health, construction industry, occupational health nursing. 


\section{RESUMO}

Objetivo: Avaliar os riscos de adoecimento presente nas atividades laborais de trabalhadores da construção civil e os fatores associados ao risco de adoecimento nessa população. Método: Trata-se de uma pesquisa transversal com abordagem quantitativa, através do Inventário sobre Trabalho e Riscos de Adoecimento. Participaram 75 trabalhadores vinculados a uma empresa do ramo de construção civil, após aprovação pelo Comitê de Ética em Pesquisa sob CAAE n. 22445413.3.0000.0118.

Resultados: Houve predomínio do sexo masculino, entre 18 a 28 anos, casados, baixa escolaridade, com 6 a 10 anos de profissão. Destaca-se os fatores Condições de Trabalho, Custo Físico e Falta de Reconhecimento, que tiveram avaliação "negativa, grave", caracterizando a produção de custo humano e sofrimento no trabalho. Os resultados apontam riscos de adoecimento entre os profissionais da construção civil.

Conclusão: Faz-se necessário rever as condições de trabalho no cenário investigado a fim de diminuir o risco de adoecimento entre trabalhadores da construção civil.

Palavras chave: Riscos ocupacionais, Saúde do trabalhador, indústria da construção civil, enfermagem do trabalho.

\section{RESUMEN}

Objetivo: Evaluar el riesgo de enfermedad presente en las actividades laborales de trabajadores de la construcción y los factores asociados con el riesgo de enfermedad en esta población. Método: Se trata de un estudio transversal con un enfoque cuantitativo a través de este riesgo y la enfermedad del Trabajo. Participaron 75 trabajadores vinculados a una empresa en el sector de la construcción, tras la aprobación del Comité Ético de Investigación bajo CAAE N. $\quad$ 22445413.3.0000.0118. Resultados: Hubo un predominio del sexo masculino entre la educación de 18 a 28 años de edad, casada, bajo, con 6-10 años de profesión. En él se destacan los factores de las condiciones de trabajo, físico y Costo Reconocimiento Lack, que tenía opinión "negativa, grave", con la producción de costo humano y el sufrimiento en el trabajo.

Resultados: Los resultados indican riesgo de enfermedad entre los profesionales de la construcción.

Conclusión: Es necesario revisar las condiciones de trabajo en la etapa de investigación con el fin de disminuir el riesgo de enfermedad entre los trabajadores de la construcción.

Palabras clave: Riesgos laborales, salud laboral, industria de la construcción, enfermería del trabajo.

\section{INTRODUÇÃO}

Nos últimos anos, o setor da construção civil é um dos que mais emprega profissionais e contribui para a economia do país de forma bastante significativa (Dieese, 2014). Embora a construção civil constitui uma das áreas mais produtivas, também é o setor que apresenta maior precariedade nas condições de trabalho e de segurança, condição agravada pela baixa escolaridade e qualificação profissional, características que tornam a construção civil um dos ramos profissionais mais arriscados em todo o mundo (Silva \& Oliveira, 2012).

As condições de trabalho nesse setor permeiam a informalidade, longas jornadas de trabalho, desproteção social, além dos salários aquém das necessidades do trabalhador e sua família (Souza, 2015). 
Inúmeros acidentes de trabalho e doenças ocupacionais advindas, sobretudo, das condições precárias de trabalho, cooperam para posicionar o setor como um dos ramos produtivos mais perigosos (Takahashi et al., 2012), potencializado pela insegurança frente ao desemprego que assombra o país.

O ritmo acelerado de trabalho, a competitividade e o modo de produção capitalista exigem constante formação e qualificação profissional do indivíduo sob pena de ficar obsoleto (Souza, 2015), o que causa tensão e estresse, que aliado a baixos salários, ambientes insalubres e de alto risco de doenças ocupacionais, influencia fortemente a saúde dos trabalhadores. Considerando os riscos ocupacionais e as condições de trabalho que são submetidos os trabalhadores da construção civil, a presente investigação propôs avaliar o risco de adoecimento dos profissionais da construção civil por meio do Inventário sobre o Trabalho e o Risco de Adoecimento (ITRA), bem como, os fatores associados a este risco.

\section{MATERIAIS E MÉTODO}

Trata-se de um estudo transversal realizado com 75 trabalhadores da construção civil num município do oeste de Santa Catarina no primeiro semestre de 2014. Foram incluídos no estudo trabalhadores com idade entre 18 e 65 anos, com tempo mínimo de seis meses de trabalho em uma empresa de construção civil, atuantes em três canteiros de obras, sendo representados por pedreiro, servente, engenheiro, mestre de obras, pintor, eletricista, encanador, azulejista e profissionais envolvidos na armação de ferro, betonagem, carpintaria e aplicação de revestimentos. A coleta de dados deu-se por meio de questionário contendo questões sociolaborais e do Inventário sobre Trabalho e Riscos de Adoecimento (ITRA), que avalia o contexto de trabalho, as exigências/custo humano no trabalho, os indicadores de prazer e sofrimento e os danos relacionados ao trabalho e foi validado no Brasil em 2003 e readaptado em 2004 e 2006 (Mendes, Ferreira \& Cruz, 2007).

O Inventário compreende escala tipo Likert de zero à cinco (0-5) pontos, sendo composto por 128 itens distribuídos em quatro escalas, a saber: Escala de avaliação do contexto de trabalho (EACT), composta por três domínios: organização do trabalho, condições de trabalho e relações socioprofissionais; Escala do custo humano no trabalho (ECHT), composta por três domínios: custo físico, custo cognitivo e custo afetivo; Escala de indicadores de prazer e sofrimento no trabalho (EIPST) com quatro domínios: Realização profissional, Liberdade de expressão, Falta de reconhecimento e Falta de liberdade de expressão; e Escala de avaliação dos danos relacionados ao trabalho (EADRT) com três domínios: danos físicos, danos psicológicos e danos sociais (Mendes, Ferreira \& Cruz, 2007).

A EACT e a ECHT seguem a classificação grave (pontuação acima de 3,7), moderado ou crítico (entre 2,3 e 3,69) e positivo ou satisfatório (abaixo de 2,3). A classificação da EIPST pode ser positiva, satisfatória (pontuação acima de 4,0), moderada ou crítica (entre 2,1 e 3,9) e grave (abaixo de 2,0), enquanto a EADRT envolve os níveis de avaliação negativa (pontuação acima de 4,1), avaliação grave (entre 3,1 e 4,0), moderado ou crítico (entre 2,0 e 3,0) e avaliação positiva, suportável (escore abaixo de 1,9) (Mendes, Ferreira \& Cruz, 2007).

A coleta de dados foi realizada em três canteiros de obras no oeste catarinense, após esclarecimento aos participantes sobre os 
objetivos do estudo e assinatura do Termo de Consentimento Livre e Esclarecido. Esta pesquisa foi aprovada pelo Comitê de Ética em Pesquisa da Universidade do Estado de Santa Catarina (Protocolo CAAE n. 22445413.3.0000.0118).

Os dados foram analisados por meio do Statistical Package for Social Sciences (SPSS), versão 20.0. Utilizou-se a estatística descritiva para análise dos dados e os valores de médias e medianas foram utilizados para a classificação dos domínios em função do estado civil, escolaridade e tempo de trabalho.

\section{RESULTADOS}

Participaram do estudo 75 trabalhadores da construção civil com idade média de $39,69 \pm 13,27$ anos, predominantemente do sexo masculino (92\%), com tempo de atuação na construção civil de 11,31 $\pm 11,17$ anos. Entre esses $43(57,3 \%)$ eram solteiros e $39(52 \%)$ haviam cursado ensino médio ou superior. A classificação dos trabalhadores da construção civil nos domínios do ITRA demonstrou que a Organização do Trabalho, Custo Cognitivo, Esgotamento Profissional, Danos Físicos e Danos Psicológicos alcançou resultado Crítico; as Relações Socioprofissionais, Custo Afetivo, Liberdade de Expressão, Realização Profissional e Danos Sociais foram classificados como Suportável; enquanto as Condições de Trabalho, Custo Físico e Falta de Reconhecimento tiveram os piores escores-Grave.

Os dados referentes à classificação dos trabalhadores da construção civil nas escalas e domínios do ITRA, em função da escolaridade e do estado civil (Tabela 1) e do tempo de trabalho (Tabela 2), são apresentados a seguir.

TABLA 1: Classificação dos trabalhadores da construção civil em função da escolaridade e estado civil. SC, Brasil, 2014

\begin{tabular}{|c|c|c|c|c|}
\hline EsCALAS/DOMÍNIOS & $\begin{array}{c}\text { ENSINO } \\
\text { FUNDAMENTAL }\end{array}$ & $\begin{array}{l}\text { ENSINO } \\
\text { MÉDIO }\end{array}$ & CASADOS & $\begin{array}{c}\text { NÃO } \\
\text { CASADOS }\end{array}$ \\
\hline \multicolumn{5}{|c|}{ Avaliação do Contexto de Trabalho (EACT) } \\
\hline Organização do Trabalho & Crítico & Grave & Grave & Crítico \\
\hline $\begin{array}{l}\text { Relações } \\
\text { Socioprofissionais }\end{array}$ & Crítico & Crítico & Satisfatório & Satisfatório \\
\hline Condições de Trabalho & Grave & Grave & Grave & Grave \\
\hline \multicolumn{5}{|c|}{ Avaliação do Custo Humano no Trabalho (ECHT) } \\
\hline Custo Afetivo & Satisfatório & Crítico & Satisfatório & Satisfatório \\
\hline Custo Cognitivo & Crítico & Crítico & Crítico & Crítico \\
\hline Custo Físico & Grave & Grave & Grave & Grave \\
\hline \multicolumn{5}{|c|}{ Avaliação de Indicadores de Prazer e Sofrimento no Trabalho (EIPST) } \\
\hline Liberdade de Expressão & Satisfatório & Crítico & Satisfatório & Satisfatório \\
\hline Realização Profissional & Satisfatório & Satisfatório & Satisfatório & Satisfatório \\
\hline Esgotamento Profissional & Crítico & Crítico & Crítico & Crítico \\
\hline Falta de Reconhecimento & Grave & Grave & Grave & Grave \\
\hline \multicolumn{5}{|c|}{ Avaliação de Danos Relacionados ao Trabalho (EDRT) } \\
\hline Danos Físicos & Crítico & Crítico & Suportável & Suportável \\
\hline Danos Sociais & Suportável & Suportável & Suportável & Suportável \\
\hline Danos Psicológicos & Suportável & Suportável & Suportável & Suportável \\
\hline
\end{tabular}

FONTE: Banco de dados dos Autores 
TABLA 2: Classificação dos trabalhadores da construção civil em função do tempo de profissão. SC, Brasil, 2014

\begin{tabular}{|c|c|c|c|}
\hline EsCALAS/DOMÍNIOS & ATÉ 5 ANOS & $\begin{array}{c}\text { DE } 5 \text { A } 10 \\
\text { ANOS }\end{array}$ & $\begin{array}{c}11 \text { ANOS OU } \\
\text { MAIS }\end{array}$ \\
\hline \multicolumn{4}{|c|}{ Avaliação do Contexto de Trabalho (EACT) } \\
\hline Organização do Trabalho & Grave & Crítico & Crítico \\
\hline $\begin{array}{l}\text { Relações } \\
\text { Socioprofissionais }\end{array}$ & Crítico & Satisfatório & Satisfatório \\
\hline Condições de Trabalho & Grave & Grave & Grave \\
\hline \multicolumn{4}{|c|}{ Avaliação do Custo Humano no Trabalho (ECHT) } \\
\hline Custo Afetivo & Satisfatório & Satisfatório & Crítico \\
\hline Custo Cognitivo & Crítico & Crítico & Crítico \\
\hline Custo Físico & Grave & Grave & Grave \\
\hline \multicolumn{4}{|c|}{ Avaliação de Indicadores de Prazer e Sofrimento no Trabalho (EIPST) } \\
\hline Liberdade de Expressão & Crítico & Satisfatório & Satisfatório \\
\hline Realização Profissional & Satisfatório & Satisfatório & Satisfatório \\
\hline Esgotamento Profissional & Crítico & Crítico & Crítico \\
\hline Falta de Reconhecimento & Grave & Grave & Grave \\
\hline \multicolumn{4}{|c|}{ Avaliação de Danos Relacionados ao Trabalho (EDRT) } \\
\hline Danos Físicos & Suportável & Suportável & Suportável \\
\hline Danos Sociais & Suportável & Suportável & Suportável \\
\hline Danos Psicológicos & Suportável & Suportável & Suportável \\
\hline
\end{tabular}

FONTE: Banco de dados dos Autores

\section{DISCUSSÃO}

Estudo identificou que os homens correspondem a 97,3\% dos trabalhadores da construção civil (Fernandes \& Vaz, 2012). No Brasil, o percentual de homens que ocupa vagas de trabalho neste segmento cresceu mais do que o de mulheres, e ainda, houve um aumento do segmento masculino contratado com carteira assinada, representando um acréscimo de mais de um milhão de postos de trabalho de homens com esse tipo de contratação totalizando $93,3 \%$. Entre as mulheres, esse aumento representou 64,0\% no período (Dieese, 2012). Observou-se nesse estudo, trabalhadores adultos jovens, reforçando achados na construção civil com prevalência entre 21 a 25 anos de 24\% (Fernandes \& Vaz,
2012) e de 23,9\% (Fernandes \& Carvalho, 2012).

Pesquisas confirmam a baixa escolaridade em trabalhadores da construção civil (Fernandes \& Vaz, 2012; Cerqueira-Santos et al., 2012), reforçando achados deste estudo. Referente carga horária semanal dos trabalhadores com carteira assinada é de 44 horas, em conformidade com a Constituição Federal Brasileira (Brasil, 1988).

\section{Avaliação do Contexto de Trabalho}

A Organização do Trabalho é definida como a divisão e conteúdo das tarefas, normas, controles e ritmos de trabalho (Mendes, Ferreira \& Cruz, 2007). Esta estrutura organizacional define como as tarefas dentro das empresas, serão alocadas, 
quem se reporta a quem e quais os mecanismos formais de coordenação e de interação que devem ser utilizados. Nesse domínio encontrou-se avaliação Moderada, Crítica, resultado mediano que representa situação-limite, potencializando o custo negativo e o sofrimento no trabalho (Mendes, Ferreira \& Cruz, 2007). Assim, a melhoria das condições de trabalho na construção civil está intrinsecamente ligada ao conhecimento das normas de segurança e sua fiscalização pelas partes envolvidas (Brasil, [s. d.]).

As Relações Socioprofissionais são definidas como os modos de gestão do trabalho, comunicação e interação profissional (Mendes, Ferreira \& Cruz, 2007), e teve avaliação Positiva, Satisfatória, resultado produtor de prazer no trabalho, aspecto que deve ser mantido e consolidado no ambiente organizacional (Mendes, Ferreira \& Cruz, 2007). Um dos fatores agravantes para o sofrimento provocado pelo trabalho é a falta de reconhecimento do trabalhador, devido a baixa qualificação e por ser facilmente substituído (Bittencourt, 2011).

As Condições de Trabalho se caracteriza pela qualidade do ambiente físico, posto de trabalho, equipamentos e materiais disponibilizados para a execução do trabalho (Mendes, Ferreira \& Cruz, 2007) e recebeu avaliação Grave, aspecto que representa risco de adoecimento para os profissionais.

No que diz respeito às condições de trabalho, o ramo da construção civil é considerado um dos mais perigosos em todo o mundo, principalmente no Brasil, liderando as taxas de acidentes de trabalho fatais, não fatais e anos de vida perdidos. Apesar dos trabalhadores dessa ocupação percebem o acidente como um acontecimento causado pelo descuido ou desatenção do trabalhador, eles também associam isso às condições de trabalho, mas especificamente a ausência de medidas e equipamentos de proteção (Iriart et al., 2008). As precárias condições de trabalho, em muitos casos, subumanas e nocivas à saúde do trabalhador, com risco constante de acidentes e de doenças, a inconstância de emprego e os baixos salários (Bittencourt, 2011), são elementos que contribuem para o aumento de exposição do trabalhador ao risco de adoecimento laboral.

Nesse contexto, além de conhecer os riscos a que está exposto no ambiente de trabalho, o trabalhador precisa conhecer a legislação trabalhista para identificar seus direitos e deveres, e para lutar por condições e segurança no trabalho (Galom, Marziale \& Souza, 2011).

\section{Avaliação de Custo Humano no Trabalho}

O Custo Físico é definido como consumo fisiológico e biomecânico imposto ao trabalhador pelas características do contexto de produção (Mendes, Ferreira \& Cruz, 2007), o qual obteve avaliação Negativa, Grave. Esse resultado é produtor de custo humano e sofrimento no trabalho, indicando forte risco de adoecimento, requerendo providências imediatas nas causas, visando a eliminação e/ou diminuição das mesmas.

O trabalho na construção civil é pesado, exige esforço e carregamento de peso, gerando sobrecarga nas vértebras, discos intervertebrais, pernas e braços (Takahashi et al., 2012). Estudo com trabalhadores da construção civil constatou que a maior parte dos movimentos realizados nas atividades laborais foram com o tronco inclinado $\mathrm{e}$ torcido, braços com movimentos abaixo dos ombros, prevalecendo o movimento em pé com ambas as pernas esticadas (Onuka, 2011), o que reforça os achados deste estudo. 
O Custo Cognitivo representa o consumo intelectual para aprendizagem, resolução de problemas e tomada de decisão no trabalho e neste estudo a avaliação foi Moderada, Crítica, resultado mediano que representa situação-limite, potencializa o custo negativo e o sofrimento no trabalho (Mendes, Ferreira \& Cruz, 2007). O trabalho pode levar ao desgaste psicológico, por que o trabalho possui aspectos negativos que incidem tanto sobre o corpo físico como a mente do indivíduo (Barros \& Mendes, 2003).

O Custo Afetivo, definido como consumo emocional, sob a forma de reações afetivas, sentimentos e de situações de humor (Mendes, Ferreira \& Cruz, 2007), obteve avaliação Positiva, Satisfatória, sendo produtor de prazer no trabalho. Esta percepção pode estar relacionada ao vinculo de trabalho dos respondentes, pois se trata de um grupo que além de ter um trabalho, este é formal e amparado pela Legislação Trabalhista.

\section{Indicadores de Prazer e Sofrimento no Trabalho}

A Escala de Indicadores de Prazer e Sofrimento no Trabalho-EIPST é composta por quatro fatores: dois para avaliar o prazer -realização profissional e liberdade de expressão, e dois para avaliar o sofrimento no trabalho- esgotamento profissional e falta de reconhecimento. A Realização Profissional constitui a vivência de gratificação profissional, orgulho e identificação com o trabalho que faz (Mendes, Ferreira \& Cruz, 2007) e, neste estudo obteve avaliação Positiva, Satisfatória.

O homem é um ser social e relacional que toma parte ativa nas relações, uma vez que se relaciona com outros trabalhadores para construir estratégias defensivas com seus pares, na tentativa de reconhecimento de sua originalidade e identidade; com a hierarquia para fazer reconhecer a utilidade de sua habilidade e de seus achados; e com os subordinados, na tentativa de buscar o reconhecimento de sua autoridade. Para identificar um bom nível de bem-estar em um trabalhador, é necessário que o mesmo demonstre ou diga estar satisfeito com seu trabalho, que identifique a existência de envolvimento com suas tarefas no contexto laboral e que estabeleça um compromisso afetivo com a organização (Ribeiro et al., 2011).

A Liberdade de Expressão representa a liberdade para pensar, organizar e falar sobre o seu trabalho (Mendes, Ferreira \& Cruz, 2007) e foi avaliada como Positiva, Satisfatória.

O Esgotamento Profissional avalia o sofrimento, que é a vivência de frustração, insegurança, inutilidade, desgaste e estresse no trabalho (Mendes, Ferreira \& Cruz, 2007), o qual obteve avaliação Moderada, Crítica, resultado que representa situação-limite.

Nesse sentido, estudo relacionado ao prazer e sofrimento indica que o trabalho pode ser prazeroso, quando as condições e o ambiente laboral forem adequados, permitindo a compatibilidade entre as exigências e capacidade do trabalhador, pois se o trabalho exigir do indivíduo mais do que pode desenvolver poderá gerar sofrimento (Barros \& Mendes, 2003). Ainda, a organização do trabalho permite $o$ trabalhador expressar sua individualidade e subjetividade, o que propicia a ressignificação do sentido do trabalho, possibilitando transformar situações de desgaste e sofrimento em situações de reconhecimento e prazer (Barros \& Mendes, 2003).

A Falta de Reconhecimento, definida como a vivência de injustiça, indignação e 
desvalorização pelo falta de reconhecimento do seu trabalho (Mendes, Ferreira \& Cruz, 2007), teve avaliação Negativa, Grave, indicando forte risco de adoecimento. $\mathrm{O}$ reconhecimento é sinalizado como o núcleo do prazer e sofrimento no trabalho, sendo que a falta deste tem sido associada a processos de sofrimento, adoecimento e despersonalização (Bendassolli, 2012).

\section{Avaliação dos Danos Relacionados ao Trabalho}

O Dano Físico entendido como dores no corpo e distúrbios biológicos (Mendes, Ferreira \& Cruz, 2007) obteve avaliação Positiva, Suportável, mesmo resultado encontrado com profissionais das áreas compartilhadas das Entidades Nacionais do Sistema Indústria (Silva, 2008).

O Dano Social se refere ao isolamento e dificuldades nas relações familiares e sociais; o psicossocial à relação do sujeito com o outro (Mendes, Ferreira \& Cruz, 2007), aspectos que tiveram avaliação Positiva, Suportável neste estudo. Com o tempo, os trabalhadores desenvolvem competências técnicas e uma série de competências relativas ao trabalho coletivo e à vida social no meio laboral (Takahashi et al., 2012), o que pode ter contribuído para avaliação positiva destes trabalhadores.

Os Danos Psicológicos são representados por sentimentos negativos em relação a si e à vida em geral (Mendes, Ferreira \& Cruz, 2007) e receberam avaliação Positiva, Suportável. O trabalho ocupa posição importante na vida das pessoas e carrega consigo valores essenciais que permeiam várias instâncias do seu viver, além disso, o trabalho exerce influência sobre a motivação e a satisfação, fator relevante para a inserção social do sujeito e na formação de sua identidade (Ribeiro et al., 2011).

Diante do exposto, é relevante apontar os domínios que se destacaram negativamente nesta pesquisa: Condições do Trabalho, indicando que os profissionais da construção civil não estão satisfeitos com as condições em seu local de trabalho; Custo Físico, indicando sobrecarga para os profissionais, resultando em custo humano e sofrimento no trabalho e; Falta de Reconhecimento, expressa pela vivência de desvalorização em função da falta de reconhecimento do seu trabalho, resultados que podem impactar no risco de adoecimento entre os trabalhadores da construção civil.

\section{CONCLUSÃO}

A indústria da construção civil é setor caracterizado como precário e que oferece risco para seus trabalhadores, tanto no âmbito de acidentes quanto nas doenças decorrentes do trabalho. O presente estudo que buscou identificar os fatores de risco ao adoecimento relacionado ao trabalho de profissionais da construção civil e identificou que os trabalhadores vivenciam um misto de prazer e sofrimento em seu cotidiano laboral.

Observou-se que os trabalhadores da amostra estão insatisfeitos com as condições de trabalho e a falta de reconhecimento e percebem alto custo físico no trabalho da construção civil. Entretanto, observa-se também, aspectos relacionais positivos no grupo, que apesar das condições negativas no trabalho, percebem relações socioprofissionais satisfatórias, liberdade de expressão, realização profissional, baixo custo afetivo e não apresentam danos sociais.

Dentre as limitações deste estudo, salientase o pequeno numero de participantes, o que inviabilizou maiores análises e também a escassez de pesquisas utilizando o 
Inventário sobre Trabalho e Risco de Adoecimento em trabalhadores da construção civil, limitando a comparação de dados e aprofundamento das discussões. Os achados sinalizam para necessidade de acompanhamento das condições de trabalho e saúde dos trabalhadores da indústria da construção civil, abre caminhos para novos estudos com essa população, bem como sugere investimentos na reestruturação organizacional e nas condições de trabalho, na consolidação das boas relações de trabalho e no desenvolvimento de estratégias que visem minimizar os riscos de adoecimento, sobretudo, os riscos físicos.

\section{REFERÊNCIAS}

- Barros, P. C. R. \& Mendes, A. M. B. (2003). Sofrimento psíquico no trabalho e estratégias defensivas dos operários terceirizados da construção civil. Psico-USF, 8 (1), 63-70. Recuperado de http://www.scielo.br/pdf/pusf/v8n1/v8n1a09.pdf.

- Bendassolli, P. F. (2012). Reconhecimento no trabalho: perspectivas e questões contemporâneas. Psicologia em Estudo, 17 (1), 37-46. Recuperado de http://www.scielo.br/pdf/pe/v17n1/v17n1a04.pdf.

- Bittencourt, N. F. B. (2011). O estresse no setor da construção civil. Profiscientia. 6,111-131. Recuperado de http://www.profiscientia.ifmt.edu.br/profiscientia/ index.php/profiscientia/article/view/70/73.

- Brasil (1998). Presidência da República. Constituição da República Federativa do Brasil de 1988. Brasília-DF: Senado Federal. Recuperado de http://www.planalto.gov.br/ccivil 03/constituicao/ constituicaocompilado.htm.

- Brasil [s.d.]. Ministério Público do Trabalho. Procuradoria Geral do Trabalho da 1. ${ }^{a}$ Região. Trabalho na Construção Civil: Direitos do empregado, deveres do empregador e principais normas de saúde e segurança na construção de obras. Brasília-DF: Senado Federal Recuperado de http://contricom.org.br/novoportal/images/arquiv ospdf/Cartilha_da_Construcao_Civil_o_Ministerio _Publico_do_trabalho.pdf.

- Cerqueira-Santos, E., Souza, D. A., Melo Neto, O. C. \& Rocha, A. C. (2012). Sexualidade do trabalhador da construção civil: percepções sobre a exploração sexual de crianças e adolescentes. Psicologia: Reflexão e Crítica. 25 (3), 578-587. Recuperado de http://www.scielo.br/pdf/prc/v25n3/v25n3a18.pdf. - DIEESE (2012). Perfil dos trabalhadores da construção civil no estado da Bahia. São Paulo: DIEESEDepartamento Intersindical de Estatística e Estudos Socioeconômicos. Recuperado de www.dieese.org.br. Recuperado de http://www.dieese.org.br/projetos/informalidade/ perfilConstrucaoCivilBA.pdf.

- DIEESE (2014). Rotatividade Setorial: dados e diretrizes para a ação sindical. São Paulo: DIEESEDepartamento Intersindical de Estatística e Estudos Socioeconômicos. Recuperado de www.dieese.org.br.

- Fernandes, A. C. P. \& Carvalho, C. M. (2012). Análise do índice de audição de trabalhadores de uma empresa de construção civil. Revista do Instituto de Ciências da Saúde. 29 (3), 231-234. Recuperado de http://www.unip.br/comunicacao/publicacoes/ics/ edicoes/2012/03 julset/V30 n3 2012 p231a234.pdf.

- Fernandes, A. C. P. \& Vaz, A. B. (2012). Perfil do índice de massa corporal de trabalhadores de uma empresa de construção civil. Revista do Instituto de Ciências da Saúde. 29 (2), 144-149. Recuperado de http://www.unip.br/comunicacao/publicacoes/ics/ edicoes/2012/02 abr-jun/V30 n2 2012 p144149.pdf.

- Galon, T., Marziale, M. H. P. \& Souza, W. L. (2011). A legislação brasileira e as recomendações internacionais sobre a exposição ocupacional aos agentes. Revista Brasileira de Enfermagem. 64 (1), 160167. Recuperado de http://www.scielo.br/pdf/reben/v64n1/v64n1a23.p df.

- Iriart, J. A. B., Oliveira, R. P., Xavier, S. S., Costa, M. A. S., Araújo, G. R. \& Santana, V. S. (2008). Representações do trabalho informal e dos riscos à saúde entre trabalhadoras domésticas e trabalhadores da construção civil. Ciência e Saúde Coletiva. 13 (1), 165-174. Recuperado de http://www.scielo.br/pdf/csc/v13n1/20.pdf.

- Mendes, A. M., Ferreira, M. C. \& Cruz, R. M. (2007). Inventário sobre Trabalho e Riscos de Adoecimento-ITRA: Instrumento auxiliar de diagnóstico de indicadores críticos no trabalho. En Mendes, AM (Ed.). Psicodinâmica do Trabalho: teoria, método e pesquisas. São Paulo: Casa do Psicólogo, 111-126.

- Onuka, F., Arantes, D. F., Andrade, F. C. \& Catai, R. E. (2011). Análise ergonômica postural do posto 


\section{Cultura de los Cuidados}

de trabalho do servente na construção civil. VII Congresso Nacional de Excelência em Gestão. Recuperado de http://www.inovarse.org/filebrowser/download/8 $\underline{543}$.

- Ribeiro, A. C. A., Mattos, B. M., Antonelli, C. S., Canêo, L. C. \& Goulard Júnior, E. (2011). Resiliência no trabalho contemporâneo: promoção e/ou desgaste da saúde mental. Psicologia em Estudo. 16 (4), 623-633. Recuperado de http://www.scielo.br/pdf/pe/v16n4/a13v16n4.pdf.

- Silva, L. S. M. T. (2008). Fatores de adoecimento nas áreas compartilhadas do Sistema Indústria. Monografia (Especialização em Gestão de Pessoas). Universidad de Brasilia: Faculdade de Economia, Administração, Contabilidade e Ciência da Informação e Documentação (FACE).

- Silva, T. F. \& Oliveira, I. R. S. (2012). A saúde do trabalhador da construção civil: o papel do enfermeiro do trabalho na prevenção de acidentes.
Recuperado

de

http://www.redentor.inf.br/arquivos/pos/publicac oes $/ 10082012$ TCC $\% 20$ TERESA $\% 20$ DE\%20FATIM A.pdf.

- Souza, R. S. (2015). Relações de trabalho e o processo de precariedade na construção civil brasileira. Estudos do Trabalho. 9 (17), 101-122. Recuperado de http://www.estudosdotrabalho.org/A6\%20RRET1 7.pdf.

- Takahashi, M. A. B. C., Silva, R. C., Lacorte, L. E. C., Ceverny, G. C. O. \& Vilele, R. A. G. (2012) Precarização do Trabalho e Risco de Acidentes na construção civil: um estudo com base na Análise Coletiva do Trabalho (ACT). Saúde e Sociedade. 21 (4), 976-988. Recuperado de http://www.scielo.br/scielo.php?script=sci arttext \&pid=S0104-12902012000400015. 